\title{
Tarifpolitischer Halbjahresbericht - Eine Zwischenbilanz der Lohn- und Gehaltsrunde 2007
}

Ein widersprüchliches Bild kennzeichnet die tarifpolitische Entwicklung im ersten Halbjahr 2007. Einerseits gelang den Gewerkschaften die Durchsetzung von Tarifabschlüssen mit deutlichen Reallohnsteigerungen. In der chemischen Industrie sowie in der Metall- und Elektroindustrie konnten die Gewerkschaften an die positive Entwicklung des Vorjahres anknüpfen und Tarifabschlüsse durchsetzen, die den gesamtwirtschaftlichen Verteilungsspielraum weitgehend ausschöpften. Dies schlug sich auch positiv in anderen Tarifabschlüssen nieder. Die Tarifwende vollzieht sich allerdings nicht ohne harte Konflikte: Das zeigen insbesondere die Arbeitskämpfe im Bauhauptgewerbe und bei der Deutschen Bahn wie auch die umfangreichen Warnstreiks, die den Abschlüssen in der Metallindustrie und in der Druckindustrie vorausgingen. In manchen Branchen mussten die Gewerkschaften auch deutlich niedrigere Abschlüsse hinnehmen. Der Konflikt bei der Deutschen Telekom AG belegt, dass in manchen Bereichen selbst in Zeiten des Aufschwungs bestehende Tarifstandards unter starken Druck geraten.

\section{Rahmenbedingungen}

Die ökonomischen Rahmenbedingungen der Tarifrunde entwickelten sich sehr positiv. Zu Jahresbeginn konstatierte das Statistische Bundesamt für 2006 ein kräftiges Wachstum von 2,5\%, das den Prognosen der Mehrheit der Forschungsinstitute zufolge in diesem Jahr noch zulegen und auch im nächsten Jahr anhalten soll (vgl. z. B. IMK 2007). Der Arbeitsmarkt belebte sich überraschend stark, die Unternehmensgewinne sprudelten weiter kräftig, sodass sich das Druckpotenzial der Gewerkschaften in dieser Tarifrunde deutlich erhöhte. Unterstützung für die gewerkschaftlichen Forderungen gab es auch im politischen Raum. Die Europäische Zentralbank (EZB) und einige Wirtschaftsinstitute wiederum formulierten die erwartbaren Warnungen vor „überzogenen“ Lohnabschlüssen, sie konnten aber die bei den Beschäftigten und in der Öffentlichkeit weitverbreitete Erwartungshaltung, dass nach Jahren mäßiger Lohnabschlüsse nun die Zeit für eine spürbare Reallohnerhöhung gekommen sei, nicht beeinflussen.

Die Lohn- und Gehaltsforderungen der Gewerkschaften fielen in dieser Tarifrunde höher aus als im Vorjahr. An der Spitze stand die Gewerkschaft Transnet mit einer Tarifforderung von 7,0 \% für die Beschäftigten der Deutschen Bahn AG. Die IG Metall forderte für ihre Hauptbranche, die

\begin{tabular}{|c|c|}
\hline \multicolumn{2}{|c|}{$\begin{array}{l}\text { Übersicht 1: Tarifforderungen in der Tarifrunde } 2007 \text { in ausgewählten } \\
\text { Tarifbereichen - in \% - }\end{array}$} \\
\hline Bauhauptgewerbe & 5,5 \\
\hline Chemische Industrie & nicht beziffert \\
\hline Deutsche Bahn AG & 7,0 mindestens $150 € /$ Mon. \\
\hline Druckindustrie & 6,5 \\
\hline Einzelhandel Nordrhein-Westfalen & 4,5 Mindesteinkommen $1.500 € /$ Mon. \\
\hline Energiewirtschaft Ost (AVEU) & 6,0/6,4 (ver.di/IG BCE) \\
\hline Groß- und Außenhandel Nordrhein-Westfalen & 6,0 mindestens $125 € /$ Mon. \\
\hline Holz und Kunststoff verarbeitende Industrie & 6,5 \\
\hline Kfz-Gewerbe Nordrhein-Westfalen & 5,0 \\
\hline Metallindustrie & 6,5 \\
\hline Nahrung-Genuss-Gaststätten & $4,0-5,5$ \\
\hline Schrott- und Recyclingwirtschaft & 7,0 \\
\hline Textilindustrie Ost & 5,0 \\
\hline Versicherungen (Innendienst) & 6,5 \\
\hline Zeitarbeit (iGZ) & $60 €$ vorweg und 5,0 \\
\hline Quelle: WSI-Tarifarchiv. & WSI MITTEILUNGEN \\
\hline
\end{tabular}

Metall- und Elektroindustrie, ein Tarifplus von 6,5 \%. Dieselbe Forderung wurde auch in der Holz- und Kunststoffindustrie und im Druckgewerbe aufgestellt. In anderen Branchen blieben die Tarifforderungen mit 4,5 bis 5,5\% zum Teil deutlich darunter (Übersicht 1).

Den ersten großen Abschluss gab es am 8.3.2007 in der chemischen Industrie (vgl. hierzu und im Folgenden Übersicht 2). Nach einer Pauschalzahlung von $70 €$ folgt eine Tarifanhebung um 3,6\% sowie eine zusätzliche Einmalzahlung von 0,7 \% eines Monatsentgelts jeweils für 13 Monate (Abschnitt 3.1). Rund zwei Monate später, am 4.5.2007, folgte der die Tarifrunde prägende Pilotabschluss in der baden-württembergischen Metallindustrie. Er sieht eine
Pauschalzahlung von insgesamt $400 €$ für die beiden ersten Monate (April und Mai) sowie eine Tariferhöhung von $4,1 \%$ ab dem 1.6.2007 vor. Ein Jahr später folgt eine Stufenerhöhung von 1,7 \% mit einer Laufzeit bis Ende Oktober 2008. Zusätzlich gibt es für Juni bis Oktober 2008 eine Einmalzahlung von jeweils $0,7 \%$ (Abschnitt 3.2).

Reinhard Bispinck, Dr., Wissenschaftler im WSI und Leiter des WSI-Tarifarchivs in der Hans-Böckler-Stiftung. e-mail: Reinhard-Bispinck@boeckler.de Götz Bauer, Monika Müller, Ulrich Schmidt, Monika Schwacke-Pilger, Andrea Taube und Monika Wiebel sind SachbearbeiterInnen im WSI-Tarifarchiv. 
Im Bauhauptgewerbe wurde eine erste Einigung von den ostdeutschen Verbänden nicht akzeptiert. Das Ergebnis der anschließenden Schlichtung wurde von den regionalen Arbeitgeberverbänden des Baugewerbes in Niedersachsen und Schleswig-Holstein gekippt, sodass die IG BAU nach einer erfolgreichen Urabstimmung in diesen Bereichen zum Arbeitskampf aufrief. Nach rund zwei Wochen Streik einigten sich die Tarifparteien Anfang Juli auf die Übernahme des leicht modifizierten Schlichtungsergebnisses (Abschnitt 3.3).

Bei der Deutschen Bahn AG rief die Tarifgemeinschaft von Transnet und GDBA nach einem mageren Arbeitgeberangebot zu Warnstreiks auf, die Gewerkschaft Deutscher Lokomotivführer (GDL) forderte einen gesonderten Spartentarifvertrag für das Fahrpersonal mit Gehaltssteigerungen um bis $\mathrm{zu} 30 \%$. Am 9.7. einigten sich Transnet/GBDA und die Deutsche Bahn auf eine Pauschalzahlung von $600 €$ für Juli bis Dezember 2007 und eine Anhebung der Tarife ab dem 1.1.2008 um 4,5\% bei einer Laufzeit bis Ende Januar 2009. Die GDL beharrte auf ihrer Forderung.

Bei der Deutschen Telekom AG musste ver.di deutliche Verschlechterungen bei den Arbeitsbedingungen für die Beschäftigten in dem neu gegründeten Bereich TService akzeptieren: Die Tarifvergütungen werden stufenweise um 6,5\% gesenkt und die Arbeitszeit wird zugleich von 34 auf 38 Stunden/Woche erhöht. Allerdings erreichte die Gewerkschaft nach langem Arbeitskampf weitreichende finanzielle Absicherungen, sodass die monatlichen Vergütungen der Beschäftigten, die zu den T-Service-Gesellschaften wechseln, zunächst gleich bleiben. Außerdem wurden ein Kündigungsschutz bis zum Jahr 2012 sowie ein Ausgründungsverzicht bis Ende 2010 vereinbart und zahlreiche tarifvertragliche Regelungen der Deutschen Telekom übernommen. Für die (verbleibenden) Beschäftigten der Deutschen Telekom werden die Vergütungstarifverträge, die Ende Juli dieses Jahres ausgelaufen wären, bis Ende 2008 unverändert verlängert.

\section{Ergebnisse im Überblick}

Im ersten Halbjahr des Jahres 2007 schlossen die Gewerkschaften des DGB Einkommenstarifverträge für rund 6,2 Mio. Be-

\begin{tabular}{|c|c|c|}
\hline \multicolumn{3}{|c|}{$\begin{array}{l}\text { Übersicht 2: Ausgewählte Lohn- und Gehaltsabschlüsse West und Ost } \\
\text { für } 2007\end{array}$} \\
\hline Abschluss & Tarifbereich & Ergebnis \\
\hline 08.03 .2007 & Chemische Industrie & $\begin{array}{l}70 € \text { Pauschale für den jew. 1. Monat } \\
3,6 \% \text { regional unterschiedlich ab } 02 / 03 / 04 / 2007 \text { für } \\
13 \text { Monate, zusätzliche Einmalzahlung von } 0,7 \% \text { eines ME, } \\
\text { multipliziert mit } 13, \text { zahlbar spätestens am } 30.06 .07\end{array}$ \\
\hline 27.03 .2007 & Textilindustrie Ost & $\begin{array}{l}\text { nach } 2 \text { Nullmonaten (April und Mai) } \\
3,0 \% \text { ab } 01.06 .07 \\
2,7 \% \text { Stufenerhöhung ab 01.07.08, Laufzeit bis } 31.03 .09\end{array}$ \\
\hline 03.04 .2007 & $\begin{array}{l}\text { Kfz-Gewerbe } \\
\text { Nordrhein-Westfalen }\end{array}$ & $\begin{array}{l}50 € \text { Pauschale für März } \\
2,5 \% \text { ab 01.04.07, Laufzeit bis } 29.02 .08\end{array}$ \\
\hline 23.04 .2007 & $\begin{array}{l}\text { Energie- und Versorgungs- } \\
\text { wirtschaft Ost (AVEU) }\end{array}$ & $3,1 \%$ ab 01.04.07, Laufzeit bis 30.04 .08 \\
\hline 04.05 .2007 & $\begin{array}{l}\text { Metallindustrie } \\
\text { (Pilotabschluss) } \\
\text { Baden-Württemberg }\end{array}$ & $\begin{array}{l}400 € \text { Pauschale insg. für April und Mai } \\
4,1 \% \text { ab } 01.06 .07 \\
1,7 \% \text { Stufenerhöhung ab 01.06.08; zusätzliche Einmal- } \\
\text { zahlung von 0,7\% eines ME (unter Berücksichtigung des } \\
\text { Urlaubsgeldes) für Juni-OKtober 2008, zahlbar im August } \\
\text { 2008, Laufzeit bis 31.10.08 }\end{array}$ \\
\hline 04.05 .2007 & $\begin{array}{l}\text { Süßwarenindustrie } \\
\text { Baden-Württemberg }\end{array}$ & $\begin{array}{l}2,4 \% \text { ab 01.07.07 } \\
2,3 \% \text { Stufenerhöhung ab 01.07.08, Laufzeit bis } 30.06 .09\end{array}$ \\
\hline 14.05.2007 & $\begin{array}{l}\text { Holz und Kunststoff } \\
\text { verarbeitende Industrie } \\
\text { Westfalen-Lippe }\end{array}$ & $\begin{array}{l}300 € \text { Pauschale insg. für Mai-Juli } \\
3,6 \% \text { ab 01.08.07 } \\
345 € \text { zusätzliche Einmalzahlung insg. für Juni-August } 2008 \\
2,5 \% \text { Stufenerhöhung ab 01.09.08, Laufzeit bis } 30.04 .09\end{array}$ \\
\hline $\begin{array}{l}15 . / 16.05 \\
2007\end{array}$ & $\begin{array}{l}\text { Papier erzeugende Industrie } \\
\text { alle West-Bereiche }\end{array}$ & $\begin{array}{l}\text { nach } 2 \text { Nullmonaten (März und April) } \\
3,2 \% \text { ab 01.05.07 } \\
2,0 \% \text { Stufenerhöhung ab 01.05.08, } 95 € \text { zusätzliche } \\
\text { Einmalzahlung im Juni 2007, Laufzeit bis } 30.09 .08\end{array}$ \\
\hline 19.05.2007 & Bauhauptgewerbe & $\begin{array}{l}\text { Schlichtungsergebnis (am 04.07. auch von den Arbeit- } \\
\text { geberverbänden Schleswig-Holstein und Niedersachsen } \\
\text { akzeptiert): } \\
\text { nach } 2 \text { Nullmonaten (April und Mai) } \\
3,1 \% \text { ab 01.06.07 } \\
1,5 \% \text { Stufenerhöhung ab 01.04.08 } \\
1,6 \% \text { Stufenerhöhung ab 01.09.08 } \\
\text { zusätzlich 0,4/0,5\% eines ME ab 01.06.07/01.04.08 } \\
\text { als mtl. Festbetrag, Laufzeit bis 31.03.09 }\end{array}$ \\
\hline 24.05 .2007 & $\begin{array}{l}\text { Hotels und Gaststätten } \\
\text { Bayern }\end{array}$ & $\begin{array}{l}\text { nach } 2 \text { Nullmonaten (April und Mai) } \\
2,2 \% \text { ab 01.06.07, Laufzeit bis } 30.04 .08\end{array}$ \\
\hline 06.06 .2007 & Druckindustrie & $\begin{array}{l}\text { nach } 3 \text { Nullmonaten (April-Juni) } \\
\mathbf{3 , 0} \% \text { ab 01.07.07 } \\
\mathbf{2 , 1} \% \text { Stufenerhöhung ab 01.07.08, Laufzeit bis } 31.03 .09\end{array}$ \\
\hline 20.06 .2007 & $\begin{array}{l}\text { Groß- und Außenhandel } \\
\text { Bayern }\end{array}$ & $\begin{array}{l}\text { nach } 3 \text { Nullmonaten (April-Juni) } \\
2,4 \%+15,50 € \text { mtl. ab 01.07.07 } \\
2,0 \% \text { Stufenerhöhung }+7,50 € \mathrm{mtl} \text {. ab 01.06.08, } \\
\text { Laufzeit bis } 31.03 .09\end{array}$ \\
\hline 20.06 .2007 & Deutsche Telekom AG & $\begin{array}{l}\text { im Rahmen des Tarifkompromisses für den Bereich T-Service: } \\
\text { Unveränderte Verlängerung der Vergütungstarifverträge der } \\
\text { Deutschen Telekom vom 01.08.2007 bis zum 31.12.2008 }\end{array}$ \\
\hline
\end{tabular}

schäftigte ab, darunter rund 0,5 Mio. in den neuen Bundesländern. Das entspricht rund $33 \%$ der von Tarifverträgen erfassten Arbeitnehmer und Arbeitnehmerinnen.

Die durchschnittliche Abschlussrate betrug für die Beschäftigten in Westdeutschland 5,0\%, für rund 0,5 Mio. Beschäftigte in Ostdeutschland 5,1 \%. Diese Größe berücksichtigt nur die tabellenwirksamen Tarifanhebungen, d.h. sie schließt auch weitere Stufenanhebungen ein, die während der Laufzeit der Verträge wirksam werden, berücksichtigt jedoch nicht Pauschal- und zusätzliche Einmalzahlungen.

Berücksichtigt man lediglich die im Jahr 2007 in Kraft tretenden Tariferhöhungen, ergibt sich ein Anstieg von 3,7\% (West: 3,7\%, Ost: 3,8\%). Differenziert man diese Größe nach Wirtschaftsbereichen, dann zeigt sich in diesem Jahr folgende Streuung: Am unteren Ende liegen der Bereich Gebietskörperschaften/Sozialversicherung mit 1,7\%, das Nahrungs- und Genussmittelgewerbe sowie der Bereich Private Dienstleistungen, Organisationen ohne Erwerbszweck mit je 2,5\%. An der Spitze stehen das Grundstoff- und Produktionsgütergewerbe mit 3,5\% und das Investitionsgütergewerbe mit 4,0\%.

Aussagekräftiger in Relation zu anderen gesamtwirtschaftlichen Größen ist die jahresbezogene Steigerung der Tarifverdienste. Sie beträgt für dieses Jahr auf der Basis der vorliegenden Abschlüsse (Stichtag 30.6.2007) durchschnittlich 2,3\%. Darin sind auch jene Steigerungen für 2007 ent- 


\begin{tabular}{lccc}
\hline Tabelle 1: Tarifsteigerung 20071) - in \% - & & \\
\hline Wirtschaftsbereich & West & Ost & Gesamt \\
\hline Gartenbau, Land- und Forstwirtschaft & 1,8 & 1,6 & 1,7 \\
Energie- und Wasserversorgung, Bergbau & 2,5 & 1,8 & 2,3 \\
Grundstoff- und Produktionsgütergewerbe & 2,6 & 3,7 & 2,7 \\
Investitionsgütergewerbe & 3,4 & 3,8 & 3,5 \\
Verbrauchsgütergewerbe & 2,5 & 1,8 & 2,4 \\
Nahrungs- und Genussmittelgewerbe & 2,1 & 2,5 & 2,2 \\
Baugewerbe & 2,5 & 2,6 & 2,5 \\
Handel & 2,1 & 2,1 & 2,1 \\
Verkehr und Nachrichtenübermittlung & 2,2 & 1,9 & 2,2 \\
Kreditinstitute, Versicherungsgewerbe & 1,8 & 1,8 & 1,8 \\
Private Dienstleistungen, Org. o. Erwerbszweck & 1,3 & 1,6 & 1,3 \\
Gebietskörperschaften, Sozialversicherung & 0,5 & 1,2 & 0,7 \\
\hline Gesamte Wirtschaft & $\mathbf{2 , 3}$ & $\mathbf{2 , 1}$ & $\mathbf{2 , 3}$ \\
\hline 1) Jahresbezogene Erhöhung 2007 gegenüber 2006. & & WSI MITTEILUNGEN \\
Quelle: WsI-Tarifarchiv 2007, Stand: 30.6.2007. & & &
\end{tabular}

halten, die bereits im vergangenen Jahr oder früher vereinbart wurden.

Diese Größe bringt - anders als die Abschlussrate-die Steigerung des durchschnittlichen tariflichen Monatsentgelts des laufenden Jahres gegenüber dem durchschnittlichen tariflichen Monatsentgelt des Vorjahres zum Ausdruck. Einbezogen werden bei dieser Größe auch die Pauschalzahlungen für verzögert in Kraft getretene Neuabschlüsse und zusätzliche Einmalzahlungen.

Die kalenderjährliche Tarifsteigerung liegt mit 2,3\% in diesem Jahr voraussichtlich deutlich über dem Wert des Vorjahres (2006: 1,5 \%). Zu berücksichtigen ist allerdings, dass große Tarifbereiche, wie z. B. der Einzelhandel mit rund 2 Mio. Beschäftigten, noch nicht enthalten sind. Zwischen den einzelnen Wirtschaftsbereichen ergeben sich deutliche Unterschiede (Tabelle 1). Angesichts einer Preissteigerungsrate von voraussichtlich unter 2,0 \% wird mit dieser durchschnittlichen Tarifsteigerung erstmals wieder eine erkennbare tarifliche Reallohnsteigerung erreicht.

Die im Vergleich zu den tariflichen Abschlussraten für 2007 geringere kalenderjährliche Steigerung hängt mit zwei Faktoren zusammen. Zum einen treten die relativ hohen Abschlüsse des ersten Halbjahres in vielen Fällen erst im Laufe des Jahres in Kraft und wirken sich demzufolge nicht auf das gesamte Jahr aus, zum anderen sind auch die Tarifabkommen des vergangenen Jahres zu berücksichtigen, die für dieses Jahr niedrige Tarifsteigerungen vorsehen. Besonders ins Gewicht fällt - wie bereits im vorigen Jahr der öffentliche Dienst, wo es infolge der Modernisierung der Tarifstrukturen (TVöD) nur Einmalzahlungen gibt. Die Spannweite zwischen den Wirtschaftsbereichen variiert in diesem Jahr ganz extrem. Am unteren En- de stehen die Dienstleistungsbereiche mit $0,7 \%$ für den Bereich Gebietskörperschaften, Sozialversicherung gefolgt vom Bereich Private Dienstleistungen, Organisationen ohne Erwerbszweck mit 1,3\%. Durchschnittlich fallen die Tarifsteigerungen für die Bereiche im Nahrungs- und Genussmittelgewerbe und Verkehr und Nachrichtenübermittlung mit 2,2 \% sowie Verbrauchsgütergewerbe mit 2,4\% aus. An der Spitze liegen die Kernbereiche der Industrie mit dem Grundstoff- und Produktionsgütergewerbe mit $2,7 \%$ und dem Investitionsgütergewerbe mit 3,5\% Tarifsteigerung.

\section{Ausgewählte Tarifrunden}

\subsection{CHEMISCHE INDUSTRIE}

Ein weiteres Mal gelang der IG BCE in diesem Jahr in ihrer Hauptbranche, der chemischen Industrie mit ihren rund $560.000 \mathrm{Be}$ schäftigten, eine zügige Tarifrunde. Erstmals gab es aber während der Verhandlungen von Teilen der mittelständischen Chemiearbeitgeber erheblichen Widerstand, sodass sich die Gewerkschaft zu größeren betrieblichen Protestaktionen und Demonstrationen veranlasst sah. Es bleibt abzuwarten, ob dies ein einmaliger Vorgang war oder ob damit ein Stück „Normalisierung“ in der chemischen Industrie eingekehrt ist, also ein Verhandlungsablauf, wie er in vielen anderen Branchen schon seit Langem üblich ist.

\section{AUSGANGSLAGE UND FORDERUNC}

Die chemische Industrie hatte in diesem Tarifjahr die tarifpolitische Vorreiterrolle inne. Mit einigem zeitlichen Abstand zur traditionellen Leitbranche Metallindustrie gingen die Tarifparteien bereits im Januar in die Verhandlungen. Die öffentliche Aufmerksamkeit war entsprechend hoch.

Mitte Oktober 2006 beschloss der Hauptvorstand der IG BCE seine Forderungsempfehlung für die Chemie-Tarifrunde 2007. Darin hieß es, der Schwerpunkt solle auf einer realen Einkommenserhöhung liegen. Des Weiteren forderte die Gewerkschaft eine Anschlussregelung für den Tarifvertrag zur Ausbildungsförderung. Ähnlich wie im Jahr 2005 verzichtete die Gewerkschaft auf eine präzise Quantifizierung der Entgeltforderung. Stattdessen hieß es lediglich, dass ein Tarifabschluss angestrebt werde, der die Produktivitätsentwicklung der Branche berücksichtige und neben dem Ausgleich der zu erwartenden Preissteigerungsrate eine reale Einkommenserhöhung sicherstelle. Das für Tarifpolitik zuständige Vorstandsmitglied Werner Bischoff konkretisierte die ökonomische Argumentation dahingehend, dass die Produktivität in der Branche im Jahr 2006 um 4,7 \% gestiegen und für 2007 eine weitere Steigerung um $3 \%$ zu erwarten sei. Die IG BCE deutete in ihrer Empfehlung bereits an, dass sie, wie bereits in früheren Abschlüssen, zu einem je nach wirtschaftlicher Situation betrieblich differenzierbaren Abschluss bereit sei.

\section{VERHANDLUNGEN UND ERGEBNIS}

Die chemische Industrie ist seit Langem bekannt für relativ kurze und geräuschlose Verhandlungen. Der Verhandlungsrhythmus in der Branche hat sich in den vergangenen Jahren so eingespielt, dass nach einer Auftaktrunde in den regionalen Tarifgebieten bundesweit weiterverhandelt wird. So geschah es auch in diesem Jahr: Die Tarifrunde startete am 15.1. mit Verhandlungen für den Tarifbezirk Nordrhein und endete Anfang Februar mit Verhandlungen für die Bezirke Saarland und Berlin. Am 8.2. fand die erste bundesweite Verhandlung statt. IG BCE-Vorstand Bischoff machte im Vorfeld deutlich, dass nach zwei Verhandlungsrunden ein Ergebnis erzielt werden sollte. Der Bundesarbeitgeberverband Chemie (BAVC) erklärte, dass angesichts rückläufiger Wachstumsraten und Produktivitätszuwächse in der Branche auch die Tariferhöhung niedriger als im Vorjahr ausfallen müsse. In den Verhandlungen selbst traten überraschend deutliche Meinungsverschie- 
denheiten zutage. Die Arbeitgeber legten deswegen kein beziffertes Angebot vor. Die IG BCE beklagte den „Realitätsverlust“ bei den Arbeitgebern, die sich in den Verhandlungen von Positionen einer möglichen Lösung wieder entfernt hätten. Nach Gewerkschaftsangaben stellten kleinere und mittlere Unternehmen den Flächentarifvertrag infrage. Offensichtlich, so IG BCE-Vorsitzender Schmoldt, gebe es eine neue Generation von Verantwortlichen, die den Wert der Sozialpartnerschaft nicht verstanden hätte. Die Gewerkschaft kündigte an, ihre Mitglieder zu Protestaktionen zu mobilisieren. Die Wirtschaftspresse spekulierte bereits über eine „gefährdete Partnerschaft" (Handelsblatt vom 12.2.2007). Vor der zweiten Verhandlungsrunde am 8.3. fanden an zahlreichen Orten Proteste mit mehreren zehntausend Teilnehmern statt, die von den Arbeitgebern als „emotionalisierte“ Demonstrationen etikettiert wurden. Doch unmittelbar vor den Verhandlungen zeigten sich die Parteien nach intensiven Vorgesprächen wieder zuversichtlich. Bereits am Nachmittag des 8.3. konnten sie ein Ergebnis präsentieren:

Entgelt

- Zahlung einer Pauschale von $70 €$ (Auszubildende $20 €$ ) jeweils für den 1 . Monat der Laufzeit,

- Anhebung der Tarifentgelte um 3,6\%, regional unterschiedlich ab 02/03/04/2007 für 13 Monate,

- zusätzliche Einmalzahlung von 0,7 \% eines Monatsentgelts multipliziert mit 13, unter Berücksichtigung u. a. der Jahresleistung und Schichtzulagen. Dies entspricht für Beschäftigte in Normalschicht 9,8 \%, in teilkontinuierlicher Schichtarbeit 10,9 \% und in vollkontinuierlicher Schichtarbeit mit Sonn- und Feiertagsarbeit 12,8 \% eines Monatsentgelts. Auszubildende erhalten $10 \%$ einer Ausbildungsvergütung. Eine Öffnungsklausel sieht vor, dass Arbeitgeber und Betriebsrat einvernehmlich aus wirtschaftlichen Gründen Vereinbarungen über die Kürzung oder den Wegfall der Einmalzahlungen treffen können.

Ausbildung

- Verlängerung des Tarifvertrages „Zukunft durch Ausbildung “ bis ins Jahr 2010 u. a. mit folgenden Änderungen:

- Verpflichtung zu einem Angebot von 16.800 Ausbildungsplätzen insgesamt für 2007 und 2008,
- Festlegung der Ausbildungsplatzzahlen bis 2010 im Rahmen der künftigen Entgelttarifverhandlungen.

\section{Langzeitkonten}

- Die tariflichen Regelungen zu Langzeitkonten wurden dahingehend ergänzt, dass neben den bisherigen Möglichkeiten auch bis zu $10 \%$ eines tariflichen Jahresentgelts eingebracht werden können.

\section{Einstellungstarife}

- Die seit vielen Jahren bestehenden Regelungen über niedrigere Einstellungstarife bei Neueinstellungen und für Langzeitarbeitslose wurden unverändert wieder in Kraft gesetzt.

In der Bewertung des Abschlusses lagen die Tarifparteien nicht weit auseinander. Die IG BCE bezeichnete das Ergebnis als ,anständigen Kompromiss" und hob vor allem das gesamte Einkommensplus von 4,3\% hervor, dass der Branchenlage entspreche. Dabei seien die Belange der weniger gut verdienenden Unternehmen berücksichtigt worden. Arbeitgeberverband und Gewerkschaften seien ihrer „gesellschaftlichen Verantwortung" gerecht geworden. Seitens des BAVC war von einem „an den wirtschaftlichen Realitäten des Jahres 2007 orientierten zukunftsfähigen Abschluss" die Rede. Als positiv wurden die vorgeschaltete Pauschale, die 14-monatige Gesamtlaufzeit und die flexibilisierte Einmalzahlung bewertet. Die Struktur des Abschlusses sei auch konform mit den Empfehlungen des Sachverständigenrates.

Die Reaktion der anderen Arbeitgeberverbände, insbesondere von BDA und Gesamtmetall, fiel kritischer aus. In der Sorge, die IG Metall werde diesen Abschluss als Messlatte nehmen, die es zu übertreffen gelte, sprach BDA-Präsident Dieter Hundt von einer dauerhaften Belastung, die „auf andere Teile der Wirtschaft nicht übertragbar" sei.

\subsection{METALLINDUSTRIE}

Die IG Metall stellte in dieser Tarifrunde unter Beweis, dass sie - immer noch - in der Lage ist, in ihrer Kernbranche einen Tarifabschluss zu realisieren, der für ihre Mitglieder eine deutliche Reallohnsteigerung bedeutet und der eine zentrale Zielsetzung der Arbeitgeber, nämlich unterhalb des Abschlusses des Vorjahres zu bleiben, wirksam durchkreuzte. Dazu trug vor allem die hohe Mobilisierungsfähigkeit bei, wie sie in den umfangreichen Warnstreiks nach Auslaufen der Friedenspflicht zum Ausdruck kam. Allerdings bleibt die tarifpolitische „Falltür" (vgl. auch den Beitrag von Ehlscheid/Urban in diesem Heft) zu beachten: Den Metallarbeitgebern gelang durch den Einbau einer Öffnungsklausel ein weiterer kleiner Schritt auf dem Weg zur Variabilisierung der Tarifentgelte

\section{AUSGANGSLAGE UND FORDERUNG}

Die Ausgangssituation war ungewohnt: Der Tarifabschluss vom April 2006 hatte anders als in den Jahren zuvor - lediglich eine kurze Laufzeit von 13 Monaten, sodass bereits ein gutes halbes Jahr danach die Diskussionen um die neue Tarifrunde begannen.

Die Abkommen liefen Ende März 2007 aus und die Tarifverhandlungen begannen demzufolge erst einige Wochen später als in der chemischen Industrie. Dennoch bestimmte die Diskussion in dieser Branche im Vorfeld die öffentliche Auseinandersetzung um die gesamte Tarifrunde 2007. Schon im Spätherbst 2006 signalisierte die IG Metall, dass die Erwartungshaltung der Belegschaften hoch sei und die positive wirtschaftliche Lage eine höhere Tarifanhebung möglich und nötig mache. Die innergewerkschaftliche Diskussion um die mögliche Forderungshöhe für 2007 zeigte, dass sich die Vorstellungen überwiegend in einem Korridor zwischen 6 und $7 \%$ bewegten. In einzelnen Unternehmen gingen die diskutierten Forderungen darüber hinaus. Bei DaimlerChrysler war von $8 \%$, bei Porsche von 9,5\% die Rede. In der Empfehlung des IG Metall-Vorstandes vom 6.2.2007 wurde die Tarifforderung dann mit $6,5 \%$ beziffert, die am 26.2. auch zur offiziellen Tarifforderung wurde. Zusätzlich wollte die IG Metall mit den Arbeitgebern u.a. über Themen wie Altersteilzeit, Leiharbeit, eine Fachkräfteinitiative, Übernahme von Studiengebühren und ausbildungsbedingten Kosten ins Gespräch kommen.

\section{VERHANDLUNGEN UND ERGEBNIS}

Die Tarifverhandlungen auf regionaler Ebene begannen am 12.3. in NordrheinWestfalen. Die Verhandlungen wurden in der ersten Runde bestimmt vom Austausch der wirtschaftlichen Begründung der jeweiligen Position. Die IG Metall stellte die hervorragende wirtschaftliche Lage der Metallindustrie in den Vordergrund (IG 
Metall 2007). Angesichts der hohen Umsatzzuwächse, weiter zunehmenden Bestellungen, der hohen Produktivität und der sehr positiven Ertragslage der Branche („die beste seit neun Jahren“) seien die geforderten Entgelterhöhungen gerechtfertigt. Metallwirtschaftlich sei mit einem Produktivitätsanstieg von 4,5\% und einer Steigerung der Verkaufspreise um $2 \% \mathrm{zu}$ rechnen. Aus gesamtwirtschaftlicher Sicht machte die Gewerkschaft für 2007 einen neutralen Verteilungsspielraum von 4,1\% geltend, der sich aus einem voraussichtlichen Produktivitätsanstieg von 1,8 \% und einem Anstieg der Verbraucherpreise um 2,3\% zusammensetze.

Die Metallarbeitgeber argumentierten dagegen im Kern wie folgt: Die positive Entwicklung des Jahres 2006 werde sich in der Metallwirtschaft nicht in gleicher Weise fortsetzen, der neue Tarifabschluss müsse daher im Volumen deutlich unter dem des Vorjahres liegen. Sie schlugen eine Aufspaltung des Abschlusses in einen fixen, tabellenwirksamen und einen variablen Teil vor. Letzterer sollte einmalig als „Konjunkturbonus" gezahlt werden und nicht in die Vergütungstabellen eingehen. Darüber hinaus forderten sie eine variable Gestaltung der Jahressonderzahlung. Eine Orientierung am Abschluss der chemischen Industrie schlossen die Metallarbeitgeber aus.

$\mathrm{Zu}$ Beginn der zweiten Verhandlungsrunde am 27.3. in Baden-Württemberg präsentierten sie ein bundesweit abgestimmtes Angebot, dass folgende Bestandteile enthielt:

- Anhebung der Tarifvergütungen um $2,5 \%$,

- eine monatliche Einmalzahlung als „Konjunkturbonus“ bei einer Laufzeit von 12 Monaten,

- variable Gestaltung des Weihnachtsgeldes über einen Zeitraum von 3 bis 5 Jahren in einer Bandbreite von $+/-15 \%$ in Abhängigkeit von der wirtschaftlichen Lage eines Betriebes.

Die IG Metall lehnte das Angebot, das erwartungsgemäß auch in allen anderen regionalen Tarifbereichen unterbreitet wurde, rundweg ab. Die tabellenwirksame Anhebung schöpfe nicht einmal den verteilungsneutralen Spielraum aus, und der „Konjunkturbonus“ sei eine Mogelpackung, da er am Ende der Laufzeit ersatzlos wegfalle. Damit wollten die Arbeitgeber ein neues tarifpolitisches Instrument installieren, das einerseits jährlich neu verhandelt werden muss und andererseits die Beschäftigten dauerhaft von der wirtschaftlichen Entwicklung abkoppelt. Die Variabilisierung des Weihnachtsgeldes führe bei einer Ausschöpfung des Spielraums nach unten zu einer Absenkung des Jahresentgeltes um $1 \%$.

Die dritte Verhandlungsrunde, die in den wichtigen Bezirken Nordrhein-Westfalen und Baden-Württemberg zeitgleich am 16.4. stattfand, brachte keine weitere Annäherung. Im Mittelpunkt standen hier die Flexibilitätsforderungen der Arbeitgeber. Auch in der vierten Runde am 26.4. konnten in beiden Bezirken keine entscheidenden Fortschritte erzielt werden. Damit lief alles auf Warnstreiks hinaus, die unmittelbar nach Ende der Friedenspflicht am 29.4. einsetzten und innerhalb weniger Tage ein großes Ausmaß erreichten. Allein am 2. und 3.5. beteiligten sich nach Angaben der IG Metall rund 100.000 bzw. 290.000 Beschäftigte an Warnstreiks und spontanen Aktionen, am 4.5. waren es noch einmal rund 76.000. Die erneuten Verhandlungen in Baden-Württemberg begannen am 3.5. und führten nach einer langen Verhandlungsnacht am 4.5. zum Ergebnis. Dies beinhaltete folgende Elemente:

- $400 €$ Pauschale für die Monate April und Mai 2007, Auszubildende $125 €$,

- Tariferhöhung um 4,1 \% ab Juni 2007,

- Stufenerhöhung um weitere $1,7 \% \mathrm{ab}$ Juni 2008,

- Erhöhung der Ausbildungsvergütungen aufgrund der tariflich geregelten festen prozentualen Relation zum Monatsgrundlohn bzw. -grundentgelt,

- Einmalbetrag von jeweils 0,7 \% eines Monatsentgeltes für die Monate Juni bis Oktober 2008 unter Berücksichtigung des Urlaubsgeldes (= 3,98 \%), zahlbar im August 2008 ,

- Verschiebung der Stufenerhöhung und der zusätzlichen Einmalzahlung in Abhängigkeit von der wirtschaftlichen Lage des Betriebes um maximal 4 Monate durch freiwillige Betriebsvereinbarung möglich, - Laufzeit des Tarifabschlusses: 19 Monate bis 31.10 .08 .

Die relativ hohe Pauschalzahlung von $400 €$ für die ersten beiden Monate entspricht bezogen auf die Durchschnittseinkommen einer Erhöhung von $6 \%$, bei $2.500 €$ Monatsverdienst $8 \%$ und bei nied- rigen Monatseinkommen $10 \%$. Die 4,1\% für die darauf folgenden 12 Monate werden angesichts einer laufenden Preissteigerungsrate von rund $2 \% \mathrm{zu}$ einer deutlichen Realeinkommenssteigerung führen. Auch die zweite Stufe von 1,7 \% geht dauerhaft in die Tabelle ein, sodass am Ende der Laufzeit die Tabellenvergütungen um insgesamt 5,87 \% höher sein werden.

Struktur und Volumen des Abschlusses werden daher von der IG Metall insgesamt sehr positiv bewertet, weil damit der von Gesamtmetall angestrebte „doppelte Paradigmenwechsel“ abgewehrt worden sei. Allerdings ist unbezweifelbar, dass es den Metallarbeitgebern gelungen ist, ihren bereits im Abschluss 2006 erstmalig realisierten Ansatz, zumindest einzelne Bestandteile des Abschlusses variabel zu gestalten, auch dieses Mal wieder durchzusetzen. Dies bezieht sich auf die Verschiebbarkeit der Stufenerhöhung wie auch der zusätzlichen Einmalzahlung. Formal kann dies nur auf Basis einer freiwilligen Betriebsvereinbarung geschehen; tatsächlich wird - wie schon beim Abschluss 2006 - viel von der realen ökonomischen Lage der Betriebe und der Konfliktfähigkeit der Belegschaften abhängen, mit dem kleinen Unterschied, dass dieses Mal nur eine Abweichung nach unten vorgesehen ist.

Neben dem Lohn- und Gehaltsabschluss wurden weitere Vereinbarungen getroffen. Dazu zählt eine Absprache zur Weiterentwicklung der Tarifregelung zum flexiblen Übergang in die Rente. Auf zentraler Ebene sollen im Jahr 2008 Verhandlungen zwischen den Tarifparteien mit dem Ziel aufgenommen werden, möglichst bis Mitte des Jahres eine entsprechende Regelung zu vereinbaren. Dabei sollen zugleich die Anforderungen an den Gesetzgeber zur Schaffung rechtlicher Rahmenbedingungen formuliert werden.

Aus Sicht der IG Metall handelte es sich bei dem Abschluss um einen „ordentlichen Kompromiss“. Es sei gelungen „für ein reales Plus in den Portemonnaies unserer Mitglieder zu sorgen und sie am Aufschwung, den sie erarbeitet haben, dauerhaft und nicht nur einmalig zu beteiligen ", so IG Metall-Vorsitzender Peters (vgl. auch den Beitrag von Ehlscheid/Urban in diesem Heft). Gesamtmetall-Präsident Kannegießer äußerte, dass der Abschluss insgesamt durch den Rückenwind der immer stärker werdenden Konjunktur beeinflusst sei. Die spürbare Entgelterhöhung sei eine „Vorleistung der Betriebe, die nun durch 
die Leistung der Mitarbeiter gedeckt werden muss". Als wichtiges Ergebnis der Tarifverhandlungen bezeichnete er es, dass der Grundsatz der betrieblichen Differenzierung fortgeführt wurde.

\subsection{BAUHAUPTGEWERBE}

Zum zweiten Mal innerhalb von fünf Jahren sah sich die IG BAU zu einem regulären Streik in ihrer Hauptbranche, dem Bauhauptgewerbe, gezwungen. Nachdem zunächst ostdeutsche Arbeitgeberverbände ein erstes Tarifergebnis nicht akzeptierten, lehnten das niedersächsische und schleswig-holsteinische Baugewerbe das anschließend durch Schlichtung gefundene Ergebnis ab. Damit steht nicht nur der Tarifabschluss 2007, sondern im Grundsatz auch das gesamte Tarifgefüge im Bauhauptgewerbe, einschließlich des tariflichen Mindestlohnes nach dem Entsendegesetz, zur Disposition.

\section{FORDERUNGEN, VERHANDLUNGEN, ERSTES ERGEBNIS}

Die Tarifverhandlungen fanden in diesem Jahr erstmals vor einer deutlich verbesserten wirtschaftlichen Lage der Branche statt. Die harte Anpassungskrise in Ost- wie Westdeutschland, verschärft durch die Auswirkungen der restriktiven Ausgabenpolitik der Gebietskörperschaften, war zu Ende gegangen. Im Jahr 2006 waren die Bauinvestitionen real bereits wieder um 3,6\% gestiegen, auch für 2007 wurde ein weiterer Anstieg erwartet. In der Folge entwickelten sich auch die Gewinnsituation der Unternehmen und - endlich - auch die Lage auf dem Bauarbeitsmarkt wieder positiv.

In der ersten und zweiten Verhandlungsrunde am 14.2. und 2.3. präsentierten die Arbeitgeberverbände Gegenforderungen: von der Abschaffung besonderer Lohnregelungen in Hamburg und Berlin über die Ausweitung der Arbeitszeitflexibilisierung bis zu vielen weiteren Verschlechterungen in den Rahmentarifverträgen. In der dritten Runde der Tarifverhandlungen am 13.3. legten sie ein erstes Angebot vor. Im Rahmen eines Tarifabschlusses war nach Auffassung des Hauptverbandes der Deutschen Bauindustrie (HDB) nur eine Kostenbelastung von insgesamt $2 \%$ denkbar. Diese Kostenbelastung müsse die Erhöhung des Beitrags zur Zusatzversorgungskasse, die möglichen Lohnerhöhungen sowie Einmalzahlungen enthalten. Die
IG BAU lehnte das Angebot der Arbeitgeber als unzureichend ab.

Ein erstes Ergebnis erzielten die Tarifparteien in der vierten Verhandlungsrunde am 31.3. Nach über 16-stündigen Tarifverhandlungen einigten sich IG BAU und Arbeitgeber in Berlin auf eine Erhöhung der Löhne und Gehälter um insgesamt 3,5 \%. Die Erhöhung setzte sich folgendermaßen zusammen:

- Erhöhung der Tariflöhne und -gehälter und Ausbildungsvergütungen um 3,1 \% ab 1.5.2007,

- zusätzlich 0,4 \% als monatlicher Festbetrag,

- Laufzeit bis zum 31.3.2008.

Parallel zur Lohnerhöhung einigten sich die Tarifvertragsparteien auf eine Erhöhung der Beiträge zur betrieblichen Altersvorsorge um 1,2 Prozentpunkte der Bruttolohnsumme. Beschäftigte und Arbeitgeber tragen sie je zur Hälfte, die Bauarbeiter zahlen dies über eine Senkung des zusätzlichen Urlaubsgeldes auf $25 \%$. Diese Vereinbarung sollte ab 1.1. 2008 gelten.

\section{SCHLICHTUNG UND STREIK}

Anfang Mai kippten die ostdeutschen Arbeitgeber die Tarifeinigung mit der Begründung, die immer noch schwierige konjunkturelle Lage des Baugewerbes im Osten lasse derartige Einkommenssteigerungen nicht zu. Damit war der Weg in die Schlichtung vorgezeichnet. Sie wurde am 14.5. von der IG BAU angerufen und legte unter Vorsitz von Ex-Bundeswirtschaftsminister Wolfgang Clement (SPD) am 19.5. folgendes Ergebnis vor, das neben einer verzögerten Tarifanhebung in diesem Jahr eine deutlich verlängerte Laufzeit mit zwei Stufenerhöhungen in den Jahren 2008 und 2009 sowie die Einführung einer tariflichen Öffnungsklausel vorsah:

- Erhöhung der Tariflöhne und -gehälter um 3,1\% ab dem 1.6. statt wie im ersten Verhandlungsergebnis ab dem 1.5.2007,

- Stufenerhöhung um weitere $1,5 \%$ ab dem 1.4.2008,

- Stufenerhöhung um weitere 1,6 \% zum 1.9.2008,

- zusätzlich Zahlung von 0,4 bzw. 0,5 \% eines Monatsentgeltes ab 1.6.2007 bzw. 1.4.2008 als monatlicher Festbetrag,

- Laufzeit bis zum 31.3.2009,
- Öffnungsklausel: Auf Basis einer Tarifvereinbarung kann vom Tariflohn im Tarifgebiet West um bis zu $8 \%$ abgewichen werden, - Anhebung der Mindestlöhne für das Tarifgebiet West auf 10,70€ bzw.12,85€ mit Wirkung zum 1.9.2008,

- Neufestsetzung der Mindestlöhne für das Tarifgebiet Ost bis zum 31.3.2008.

Am 4.6. sorgten dann Arbeitgeber des Bauhandwerks in Niedersachsen und Schleswig-Holstein für ein Scheitern, indem sie das Schlichtungsergebnis ablehnten. Die Baukonjunktur im Norden, so ihre Argumentation, verkrafte die Höhe des Abschlusses nicht. Außerdem wollten sie die im Schlichtungsergebnis enthaltene Öffnungsklausel noch weiter an ihre Vorstellungen anpassen, sodass betriebliche Abweichungen ohne Einbeziehung der IG BAU möglich würden. Dies stieß auf heftige Kritik insbesondere des Hauptverbandes der Bauindustrie, da Vertreter dieser beiden Regionalverbände an der Aushandlung des Schlichtungsergebnisses selbst beteiligt waren.

Daraufhin begann die IG BAU mit Warnstreiks und führte eine Urabstimmung in den beiden betroffenen Tarifgebieten durch, in der sich 87,9 \% der Mitglieder für Streikmaßnahmen aussprachen. Am 18.6. traten mehrere hundert Bauarbeiter auf rund 100 Baustellen in den Streik. In den folgenden Tagen weitete die Gewerkschaft den Arbeitskampf aus. Ende Juni befanden sich nach Gewerkschaftsangaben über 1.700 Bauarbeiter auf rund 250 Baustellen im Arbeitskampf. Nach Angaben der IG BAU arbeiten im Streikgebiet insgesamt rund 42.000 Beschäftigte am Bau, davon etwa 8.000 Alleinunternehmer. Von den Beschäftigten im Bauhandwerk in Niedersachsen und Schleswig-Holstein seien 9.800 IG BAU-Mitglieder. Nach zwei Sondierungsgesprächen einigten sich die Tarifparteien auf einen Kompromiss. Danach wird der Schlichtungsspruch mit einer Änderung angenommen: Beide Seiten werden umgehend beim Bundesarbeitsminister beantragen, den Mindestlohn West noch in diesem Jahr auf $13 €$ zu erhöhen. Damit soll verhindert werden, dass sich die Schere zwischen tarifgebundenen und nicht tarifgebundenen Unternehmen weiter öffnet. Es wurde außerdem ein Verfahren vereinbart, wie die Firmentarifklausel umgesetzt werden kann, die auf Wunsch der Nordverbände in den Schlichtungsspruch aufgenommen wurde. Für die Annahme gilt eine Zustimmungsfrist bis zum 
12.7.2007. Die Streiks werden fortgeführt, bis die Zustimmung der Baugewerbeverbände Niedersachsens und Schleswig-Holsteins vorliegt und die Urabstimmung abgeschlossen ist.

\section{Offene Tarifrunden}

Die Tarifrunde 2007 ist in den großen Tarifbereichen - von wenigen Ausnahmen abgesehen - abgeschlossen: Noch nicht beendet sind die Verhandlungen im Einzelhandel. Hier gestalten sich die Tarifverhandlungen auch deswegen besonders schwierig, weil die Arbeitgeber nach der Freigabe der Ladenöffnungszeiten vor allem die Spätarbeitszuschläge streichen wollen, während ver.di verlässliche Regelungen zur Arbeitszeitgestaltung, die Einführung eines Flexibilitätszuschlages sowie die Begrenzung von prekären Beschäftigungsverhältnissen fordert. Ende August läuft der Gehaltstarifvertrag im Versicherungsge- werbe aus. Hier fordert ver.di eine Gehaltsanhebung von 6,5\% sowie Regelungen zur Qualifizierung, Beschäftigungssicherung und Verlängerung der Altersteilzeitbestimmungen. Ende des Jahres laufen die Tarifverträge im öffentlichen Dienst bei Bund und Gemeinden aus. Ver.di fordert eine ”deutliche“ Einkommenserhöhung, wobei sich die Gewerkschaft an den Abschlüssen der privaten Wirtschaft orientieren will.

\section{LITERATUR}

Bispinck, R./WSI-Tarifarchiv (2007): Tarifpolitischer Jahresbericht 2006 Aufwärtstrend bei Tarifabschlüssen - Konflikte um Beschäftigungssicherung und Tarifstandards, in: WSI-Informationen zur Tarifpolitik, Düsseldorf, Januar

IG Metall (2007): Tarifrunde 2007 in der Metallindustrie: Sozialökonomische Rahmenbedingungen, Wirtschaftspolitische Informationen 1 vom 26.2., Frankfurt/Main
Institut für Makroökonomie und Konjunkturforschung (IMK) (2007): Der Aufschwung geht weiter, Frühjahrsprognose des IMK für 2007, IMK-Report 19, April 